\title{
Purification of DP 6 to 8 chitooligosaccharides by nanofiltration from the prepared chitooligosaccharides syrup
}

\author{
Huizhong Dong ${ }^{1,2}$, Yaosong Wang ${ }^{1,2}$, Liming Zhao ${ }^{1,2^{*}}$, Jiachun Zhou ${ }^{1,2}$, Quanming Xia ${ }^{1,2}$, Lihua Jiang ${ }^{1,2}$
} and Liqiang Fan ${ }^{1,2}$

\begin{abstract}
Background: Chitooligosaccharides (COS) with degrees of polymerization (DP) 6 to 8 are degraded from chitosan, which possess excellent bioactivities. However, technologies that could purify them from hydrolysis mixtures in the narrow DP range (984 to 1,306 Da) are absent. The objective of this research is to purify DP 6 to 8 COS by nanofiltration on the basis of appropriate adjustments of the feed condition.

Methods: Syrup containing DP 6 to 8 COS at different concentrations (19.0 to $46.7 \mathrm{~g} / \mathrm{L}$ ) was prepared. A commercial membrane (QY-5-NF-1812) negatively charged was applied. Experiments were carried out in full recycle mode, so that the observed COS retentions were investigated at various transmembrane pressures (6.0 to 20.0 bar), temperatures $\left(10^{\circ} \mathrm{C}\right.$ to $50^{\circ} \mathrm{C}$ ), and pHs (5.0 to 9.0). Then, the feasibility of separation of DP 6 to 8 COS was further studied by concentration ratio under optimum conditions.

Results: The results indicate that the purification of DP 6 to 8 COS by nanofiltration NF is feasible. It was found that the permeate flux was $95.0 \mathrm{~L} /\left(\mathrm{m}^{2} \mathrm{~h}\right)$ at $10.0 \mathrm{bar}$, while it reached to $140.0 \mathrm{~L} /\left(\mathrm{m}^{2} \mathrm{~h}\right)$ at $20.0 \mathrm{bar}$, and it increased with feed temperature, but the membrane pores were also swelled by heating and led to an irreversible wastage of target oligomers. Additionally, the retention behaviors of chitooligosaccharides are significantly influenced by $\mathrm{pH}$.

Conclusions: Although glucosamine and dimer were permeatable at low pH, their retention ratios were remarkably varied from 0.458 to 0.864 when $\mathrm{pH}$ was 9.0. With the interaction of hydrogen bonds, structural curling and overlapping of chitooligosaccharides were formed. Consequently, the rejection of chitooligosaccharides at various pHs is variable. Spray-dried products were finally characterized by the matrix-assisted laser desorption/ionization time-of-flight mass spectrum. The spectrum identified the distributions of hexamer, heptamer, and octamer. Combined with high-performance liquid chromatography profiles, the purity and yield of DP 6 to 8 chitooligosaccharides were up to $82.2 \%$ and $73.9 \%$, respectively.
\end{abstract}

Keywords: Nanofiltration; Chitooligosaccharides; Degree of polymerization; Separation; MALDI-TOF-MS

\section{Background}

Chitooligosaccharides (COS) are defined as the partially degraded products of chitosan or low-molecular-weight chitosan (LMWC) with a degree of polymerization (DP) ranges from 2 to 20 [1]. As shown in Figure 1, their molecular structures are linear oligosaccharides composed

\footnotetext{
* Correspondence: zhaoliming@ecust.edu.cn

${ }^{1}$ State Key Laboratory of Bioreactor Engineering, R\&D Center of Separation and Extraction Technology in Fermentation Industry, East China University of Science and Technology, Shanghai 200237, China

${ }^{2}$ Shanghai Collaborative Innovation Center for Biomanufacturing Technology, Shanghai 200237, China
}

of 2-amino-2-deoxy-D-glucopyranose and 2-acetamino2-deoxy-D-glucopyranose (Figure 1(A)) units which are linked by $\beta(1 \rightarrow 4)$ glycosidic bonds [2].

According to many previous reports, COS possess a series of attractive bioactive properties, including antibacterial [3], anticoagulant [4], antimicrobial [5], antioxidant [6], anticancer [7], hypolipidemic [8], and immune-stimulating [9] effects. Based on these excellent advantages mentioned before, COS are responsible for practical applications in beverage processing [10], functional ingredients [11], and biomedicines [12], which are different from chitosan and

\section{实}




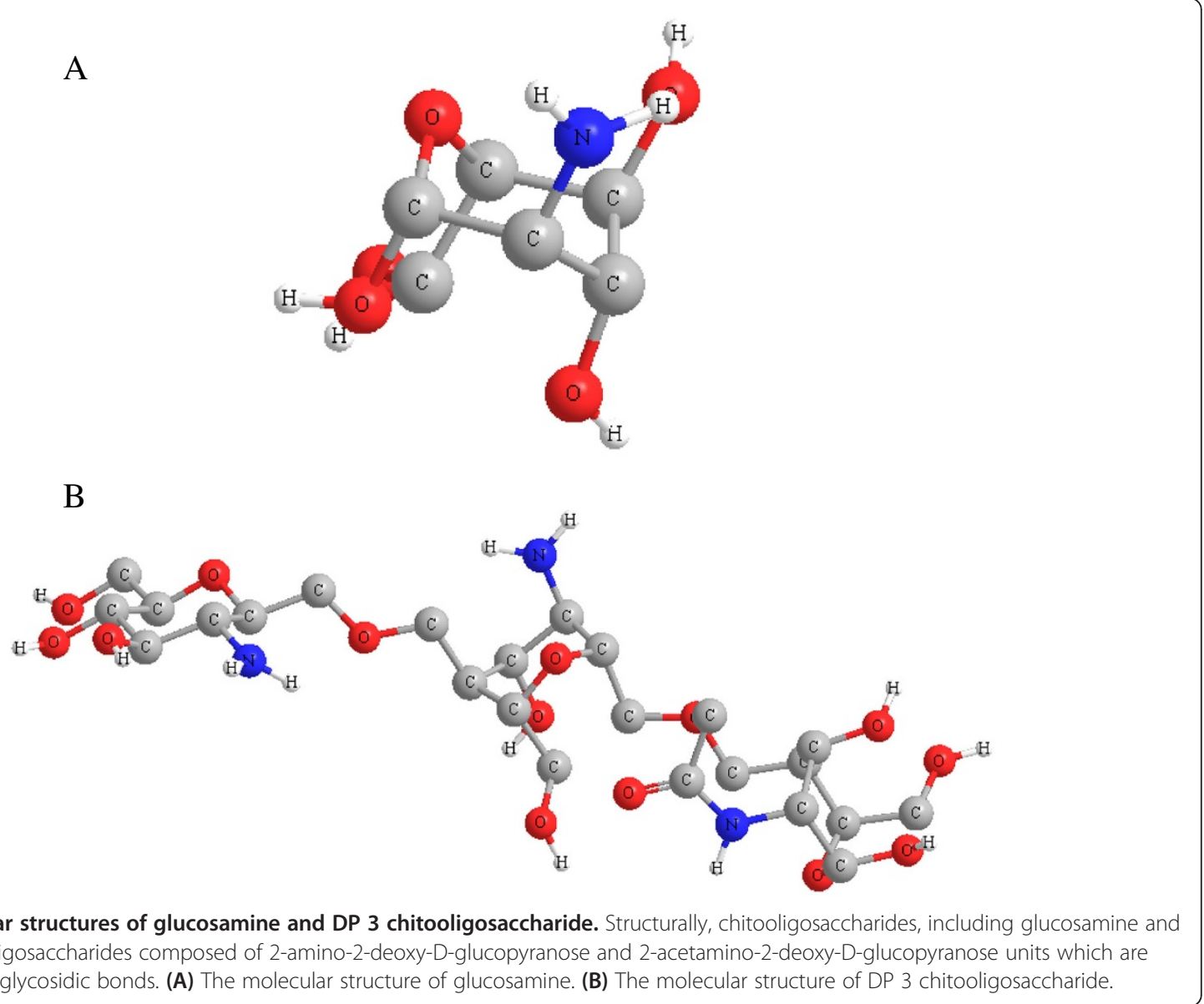

chitin except for their contributions on food packaging [13]. Nowadays, hybrid enzymatic hydrolysis has become the ideal technology for COS preparation due to its high efficiency and little structural modification [14]. Unfortunately, the products after enzymatic degradation were just intermediate ones [15]. Suitable methods should be used to separate target COS from mixtures coexisting in the solution, such as the high molecular weight of chitosan, proteins (enzymes), and inorganic salts.

Conventionally, COS is purified by various chromatographies. Fan et al. succeeded in obtaining COS by macroporous resins from fermentation broth, and the productivity of target products could go up to $90 \%(w / w)$ under optimum conditions [16]. Meanwhile, Cabrera and Custem reported that the concentrations of $\operatorname{COS}$ could be analyzed by matrix-assisted laser desorption/ionization time-of-flight mass spectrum (MALDI-TOF-MS) [17]. Although the chromatography technology is feasible to remove irrelevant components and refine COS in terms of DP, it is only accepted in laboratory analysis but far from scale-ups. In practice, several negative problems inevitably appear during continuous using of chromatography, including the demands on cleaning and regeneration of column packing, adsorption capacities of COS, and relatively high production cost $[18,19]$.

Recently, there is a growing attention on nanofiltration (NF) applications, especially for COS preparation. Kim et al. reported that several instruments were used to prepare COS, the details of which could be summarized in immobilized enzymatic columns and ultrafiltration (UF) membrane reactors [2]. Han et al. demonstrated the desalination feasibility of NF-40 membrane for chitobiose solution. Under acidic conditions, the interception of solutes ranks as chitobiose $>$ glucosamine $>\mathrm{Na}^{+}>\mathrm{H}^{+}$ [20]. Furthermore, the steric and electrostatic effects are inferred according to the sequence. Han et al. also studied the influence of three membranes (DL, DK, NTR-7540) on COS separation and drew identical conclusions [21]. In a word, NF has been proved to be an effective technology for the separation and purification of COS.

Chitosan was originally hydrolyzed by enzymes, which resulted in the coexistence of various DP COS, glucosamines, and salts. It is evident that there is a great difference in molecular weight among the solutes. Also, the wider ranges of hydrolysis products make it difficult to improve the purity and yield of COS. For instance, DP 6 
to $8 \operatorname{COS}(984$ to $1,306 \mathrm{Da}$ ) plays an important role in cancer curing [22]. Therefore, this study is to investigate the separation performance of DP 6 to 8 COS at different solution properties and present a promising purification technology by NF.

\section{Methods \\ Materials}

The raw syrup containing DP 6 to 8 COS was made from chitosan by enzymatic hydrolysis. Chitosan was supplied by Yunzhou Biochemistry Co., Ltd. (Shandong, China). The enzyme mixture (chitosanase from Streptomyces griseus - EC 3.2.1.132; cellulase from Trichoderma - EC 3.2.1.4) was obtained from Golden-Shell Biochemical Co. (Zhejiang, China). DP 2 to $8 \mathrm{COS}$ and glucosamine standards were purchased from Huicheng Biochemical Co., Ltd. (Shanghai, China). All chemicals used in the NF operation and high-performance liquid chromatography (HPLC) analysis were analytical grade or chromatographic grade, respectively. Deionized water (conductivity $<3.0$ $\mu \mathrm{s} / \mathrm{cm}$ ) for membrane cleaning was produced by ion exchange.

\section{Preparation of chitooligosaccharide syrup}

Chitosan (91.5\% degree of deacetylation) was dissolved in acetic acid $(1 \%, w / v)$ with stirring, and $\mathrm{pH}$ was adjusted to 5.3 , and then kept at $45^{\circ} \mathrm{C}$. The chitosan concentration was $5 \%(w / v)$. Combined enzymes $(75 \mathrm{U} / \mathrm{g})$ based on chitosan were added into the chitosan solution and hydrolyzed for $6 \mathrm{~h}$, and then, the hydrolysis was terminated by immersion in a boiling water bath. Finally, a UF membrane module
(QY-3-UF-1812, AMFOR Inc., Newport Beach, US) was applied to remove enzymes at $50^{\circ} \mathrm{C}$. After cooling to ambient temperature, the syrup was diluted to various concentrations for NF separation.

\section{Membrane}

Purification experiments were operated in a pilot setup (Figure 2). The setup composes of a feed tank, a high-flux pump, a membrane vessel, and a flowmeter. Moreover, the inlet and outlet pressures were metered by two pressure gauges, and the feed temperature could be regulated by a circulating cooling water system surrounding the feed tank.

The membrane module employed was an organic polymer composite with spiral-wound structures (QY-5-NF1812, AMFOR Inc., Newport Beach, US). The membrane module is measured as $1.8 \mathrm{in} .(4.6 \mathrm{~cm})$ in section diameter and 12 in. $(30.5 \mathrm{~cm})$ in length, which is so-called 1812 type module. Also, it is measured by an approximate molecular weight cut-off (MWCO) of $500 \mathrm{Da}$, which is close to the molecular weight of DP 4 COS. As described in Table 1, the membrane is negatively charged that can tolerate a maximum pressure up to 25.0 bar. The effective surface area is $0.2 \mathrm{~m}^{2}$. The temperature and $\mathrm{pH}$ tolerance range cover from $0^{\circ} \mathrm{C}$ to $60^{\circ} \mathrm{C}$ and 4 to 12 , respectively.

\section{Membrane permeate flux}

In this study, the permeate flux is represented as the average one in process. The average permeate flux $\left(J_{\mathrm{v}}\right)$ is calculated by Equation 1, as follows:

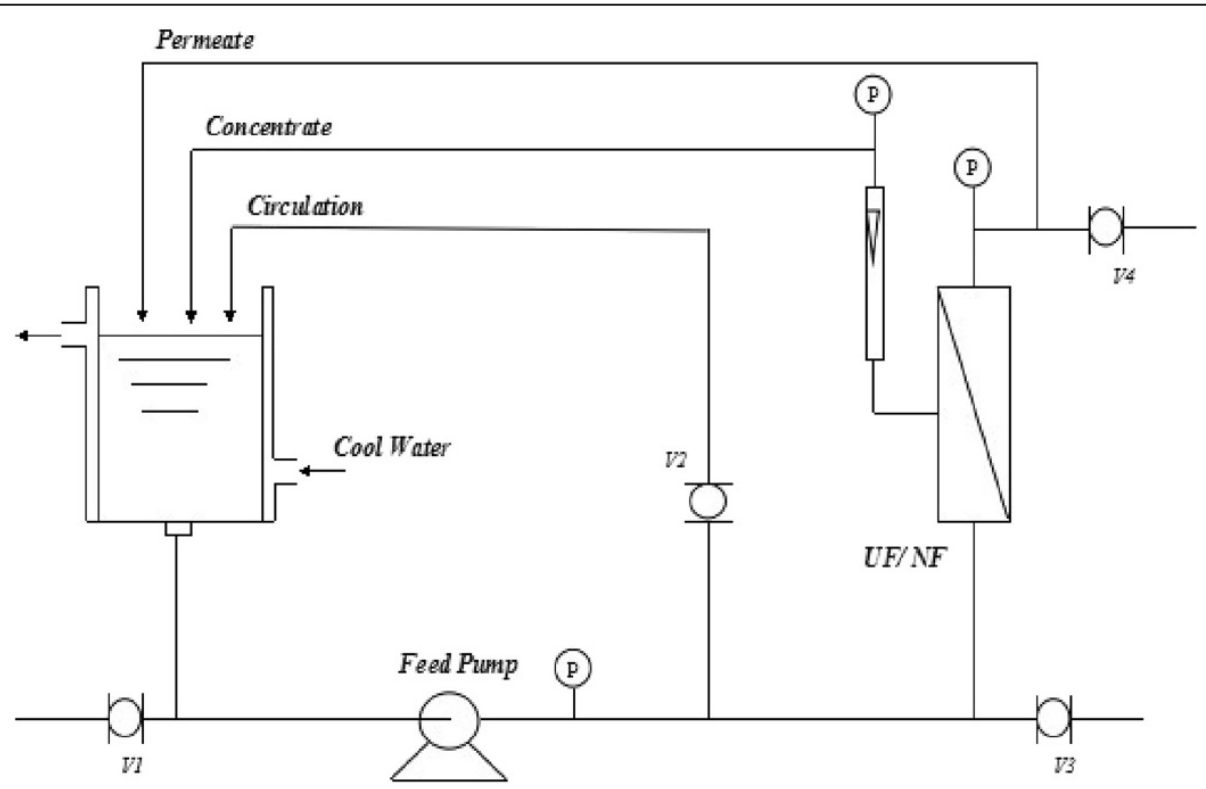

Figure 2 Brief scheme of the nanofiltration system. Purification experiments were operated in batch equipment for laboratory use. V1 to V4 were represented as valves, and $\mathrm{P}$ are pressure gages. 
Table 1 Information of NF membrane module

\begin{tabular}{lc}
\hline Membrane index & Parameter \\
\hline Type & 1812 \\
Texture & Organic polymers \\
Filtration area $\left(A_{\mathrm{m}}\right)$ & $0.2 \mathrm{~m}^{2}$ \\
MWCO & $500 \mathrm{Da}$ \\
Temperature & $0^{\circ} \mathrm{C}$ to $60^{\circ} \mathrm{C}$ \\
$\mathrm{pH}$ & 4 to 12 \\
Pressure & $<25.0 \mathrm{bar}$ \\
\hline
\end{tabular}

$$
J_{\mathrm{v}}=\frac{V_{\mathrm{p}}}{A_{\mathrm{m}} \times t}
$$

where $J_{\mathrm{v}}$ is the average permeate flux of the membrane $\left[\mathrm{L} /\left(\mathrm{m}^{2} \mathrm{~h}\right)\right] ; V_{\mathrm{p}}$ is the volume of permeate accumulated in testing time $(L) ; A_{\mathrm{m}}$ is the effective areas of membrane $\left(\mathrm{m}^{2}\right) ; t$ is the testing time $(\mathrm{h})$.

\section{Observed retention ratio}

Retention ratio is also one of the most important factors in membrane separation. The observed retention ratio is described by Equation 2:

$$
R_{\mathrm{obs}}=1-\frac{C_{\mathrm{p}}}{C_{\mathrm{b}}}
$$

where $R_{\mathrm{obs}}$ is the observed retention ratio of solute (\%); $C_{\mathrm{p}}$ is the concentration of solute in the permeate $(\mathrm{mol} / \mathrm{L}) ; C_{\mathrm{b}}$ is the concentration of solute in the feed (mol/L).

\section{Full recycle mode of NF}

Initially, 5.0 L raw syrup was added, and the recycling flow rate was adjusted to $360 \mathrm{~L} / \mathrm{h}$, which represented the optimum rotation condition for the working pump. The effects of transmembrane pressure (TMP), operation temperature, and $\mathrm{pH}$ on NF performance were successively carried out. All the permeate were flowed back to the feed tank, while the $J_{\mathrm{v}}$ and retention behaviors of DP 6 to $8 \mathrm{COS}$ at different concentrations were measured after the renewed conditions were stable for $15 \mathrm{~min}$. It is worth being noted that two of the parameters mentioned must be constant when the third is variable. During the process, the temperature was controlled at $50^{\circ} \mathrm{C} \pm 2^{\circ} \mathrm{C}$ by a heat exchanger surrounding the feed tank, except for the experiments to investigate the effects of temperature on the NF retention on DP 6 to 8 COS.

\section{Purification of DP 6 to 8 chitooligosaccharides}

Purification experiments for DP 6 to 8 COS were executed in batch mode. Under the optimized conditions obtained from the preliminary experiments, the concentrate stream was circulated back to the feed tank, whereas the permeate was collected individually. Considering the practical capacity of the feed tank, 7.0 L of diluted syrup $\left(C_{\mathrm{b}}=19.0 \mathrm{~g} / \mathrm{L}\right)$ was added firstly. Every 1.0 L of extra diluted syrup should be supplied as soon as the permeate was equally removed. Certainly, the systematic temperature during NF was maintained by cooling water. After adding all the syrup $(16.0 \mathrm{~L})$, the process was terminated until the volume of permeate reached $14.0 \mathrm{~L}$ (2.0 L syrup left in the tank). The effect of the concentration ratio on the purity of DP 6 to 8 COS was confirmed by flux and rejections.

\section{HPLC analysis}

The concentrations of glucosamine and DP 2 to 8 COS were analyzed by an HPLC system (Shimadzu 10A, Shimadzu, Kyoto, Japan) equipped with a high-perfor mance sugar column (Shodex Asahipak NH2P-50 4E, Shodex, Kyoto, Japan) and an RI detector. The mobile phase consists of methyl cyanide and pure water with the ratio of 70:30 $(v / v)$. The column temperature was maintained at $30^{\circ} \mathrm{C}$, and the flow velocity was kept at 1.0 $\mathrm{mL} / \mathrm{min}$. All the solutes were measured in the form of single-arranged peaks. In general, the glucosamine was firstly eluted, and then, the dimer and trimer were sequentially characterized due to the adsorption strength difference to the stationary phase. The distributions of COS were quantified by integrating peak areas.

\section{MALDI-TOF-MS analysis}

MALDI-TOF-MS analysis of COS was carried out using Shimadzu AXIMA Performance matrix-assisted laser desorption/ionization time-of-flight mass spectrometry (Shimadzu, Kyoto, Japan). All spectra were measured in the reflector mode by external calibration. The laser was scanned at a scale from 500 to $1,500 \mathrm{Da}$. An aqueous

Table 2 The component analysis of raw materials for the NF process

\begin{tabular}{lccc}
\hline Chitosan (\%) & Total Proteins $^{\mathbf{a}}(\mathbf{m g} / \mathbf{L})$ & Glucosamine $^{\mathbf{a}} \mathbf{( g / L )}$ & DP $\mathbf{6}{\text { to } \mathbf{8} \text { chitooligosaccharides }^{\mathbf{a}}(\mathbf{g} / \mathbf{L})}^{9.6 \pm 1.2}$ \\
\hline 1.0 & $1.4 \pm 0.3$ & $0.4 \pm 0.1$ & $19.0 \pm 1.5$ \\
2.0 & $2.7 \pm 0.2$ & $0.9 \pm 0.1$ & $28.3 \pm 1.7$ \\
3.0 & $3.3 \pm 0.4$ & $1.4 \pm 0.2$ & $37.6 \pm 2.4$ \\
4.0 & $2.9 \pm 0.3$ & $1.8 \pm 0.2$ & $46.7 \pm 2.1$ \\
5.0 & $1.4 \pm 0.3$ & $2.3 \pm 0.4$ & \\
\hline
\end{tabular}

${ }^{a}$ The data were determined by three parallel measurements. 

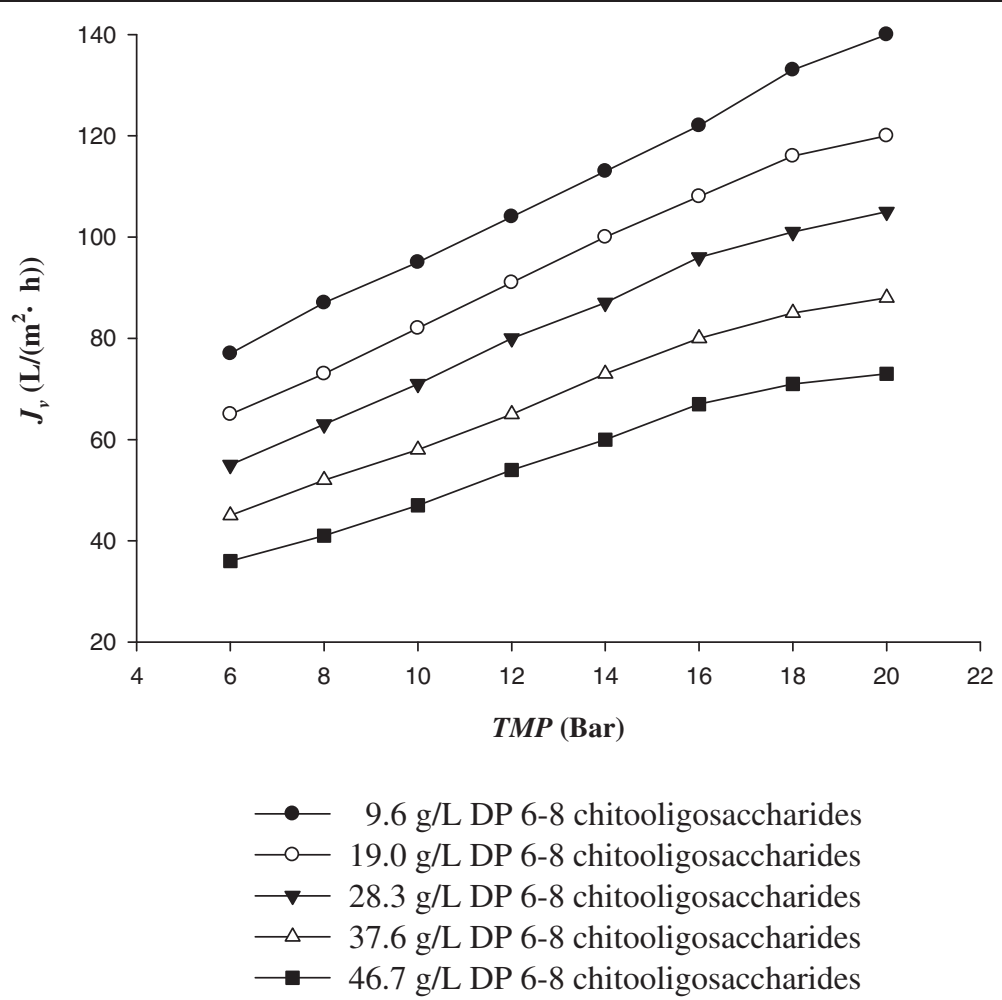

Figure $3 \mathrm{TMP}$ vs. $J_{\mathrm{v}}$ of chitooligosaccharide syrup at $50^{\circ} \mathrm{C}$ during $\mathrm{NF}$ separation. $J_{\mathrm{v}}$ of chitooligosaccharide syrup with different $C_{\text {chitosan }}$ ranged by transmembrane pressure (6.0 to 20.0 bar). The permeate fluxes increased with the pressure, whereas they decreased with the increasing concentration of DP 6 to 8 chitooligosaccharides.

solution of 2,5-dihydroxybenzoic acid (DHB, $100 \mathrm{mg} / \mathrm{mL}$ ) was used as the matrix.

\section{Statistical methods}

All parameters and experimental results were obtained with means \pm SD of parallel tests. The data was analyzed by Statistix 9.0 software (Analytical Software, Tallahassee, FL, USA).

\section{Results and discussion}

\section{Characterization of chitooligosaccharide syrup}

As the raw material for NF process, the COS syrup was defined as the permeate of UF that was pretreated after enzymatic hydrolysis. Summarized by the previous tests, $10.0 \mathrm{~L}$ of chitosan hydrolysates were prepared and purified by UF, while a $2.0-\mathrm{L}$ concentrate and $8.0-\mathrm{L}$ permeate were separately bulked at each concentration level due to the cross-flow filtration mode.

Table 2 shows that the principle components of COS syrup varied with the changeable concentrations of chitosan $(1.0 \%$ to $5.0 \%, w / w)$ after UF. It demonstrated that most of the target products permeated through the UF membrane, while the blocking layer adhered to by macromolecular solutes was slightly affected. However, there was still a little amount of proteins $\left(C_{\text {protein }}=1.4\right.$ to $\left.2.9 \mathrm{mg} / \mathrm{L}\right)$ that remained, which was partially caused by the temperature effect and concentration polarization. Besides the protein content in the purified syrup, its calculation data was in good agreement with the $R_{\text {obs }}$ during the UF process, which was scaled from 0.996 to 0.998 (the data was not shown). The phenomenon suggested that the tested UF membrane was sensitive to the protein rejections.

In addition, with the increment of crude concentration $\left(C_{\text {chitosan }}\right)$, the contents of glucosamine $\left(C_{\mathrm{s}}\right)$ and DP 6 to $8 \mathrm{COS}\left(C_{\mathrm{DP}} 6\right.$ to 8$)$ were also dramatically increased. For example, $C_{\mathrm{g}}$ and $C_{\mathrm{DP}} 6$ to 8 at $C_{\text {chitosan }}=2.0 \%$ were 0.9 and $19.0 \mathrm{~g} / \mathrm{L}$, respectively, while those at $C_{\text {chitosan }}=5.0 \%$ changed to 2.3 and $46.7 \mathrm{~g} / \mathrm{L}$, respectively. The tendency indicates that the preparation and separation process of DP 6 to 8 COS was rarely affected by the syrup concentration. According to the data listed in Table 2, the conclusion could be drawn that the purification of hydrolysates by the UF membrane was highly performed. What is more important, the excellent elimination of unexpected impurities may greatly benefit for the next NF treatments.

\section{Effects of transmembrane pressure on permeate flux}

Figure 3 illustrates that the permeate fluxes $\left(J_{\mathrm{v}}\right)$ of COS syrup at different $C_{\text {chitosan }}$ increase with the TMP from 6.0 to 20.0 bar. Moreover, it shows a linear relationship 

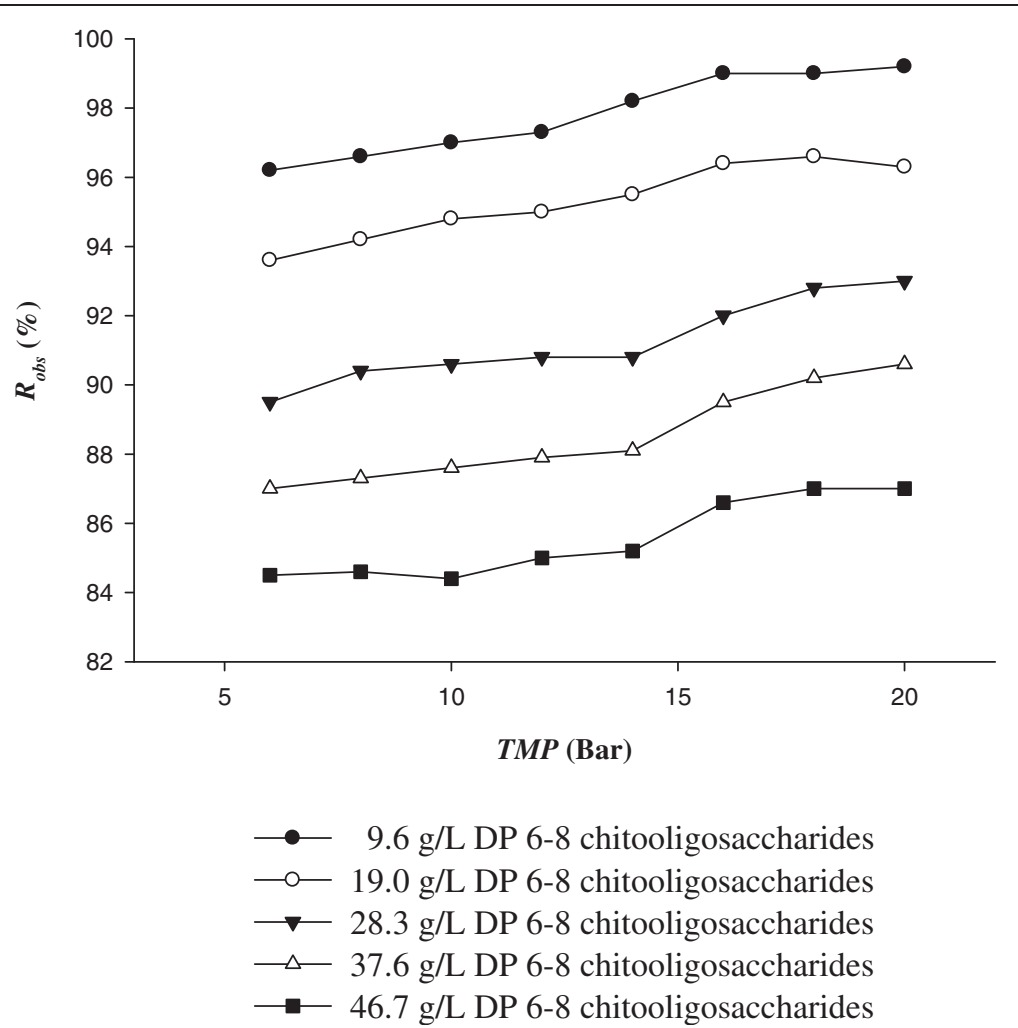

Figure 4 TMP vs. $\boldsymbol{R}_{\mathrm{obs}}$ of DP 6 to 8 chitooligosaccharides at $\mathbf{5 0}^{\circ} \mathrm{C}$ during NF separation. It pictured the retentions of DP 6 to 8 chitooligosaccharides when the TMP was set between 6.0 and 20.0 bar. The $R_{\text {obs }}$ of DP 6 to 8 chitooligosaccharides were significantly judged by viscosity and pressure applied.

between TMP and $J_{\mathrm{v}}$. For instance, the $J_{\mathrm{v}}$ was $95.0 \mathrm{~L} /\left(\mathrm{m}^{2} \mathrm{~h}\right)$ at 10.0 bar for the $9.6-\mathrm{g} / \mathrm{L}$ COS solution, whereas it elevated to 122.0 and $140.0 \mathrm{~L} /\left(\mathrm{m}^{2} \mathrm{~h}\right)$ at 16.0 and 20.0 bar at the same syrup concentration, respectively. Membrane compaction reduces the mass transfer resistance and enhances the velocity on membrane surface, and thus, leads to an increasing permeate volume of feed solution in regular intervals.

Conversely, $J_{\mathrm{v}}$ decreased with the increment of concentration of DP 6 to 8 COS. It could be well explained by the interaction of TMP and the accumulation effects. On the one hand, the solutes would be greatly accumulated by the driving pressure, which is beneficial for the formation of cake layer around the boundary of the membrane. As a result, the membrane pore would be partially blocked and result in a decrement of $J_{\mathrm{v}}$. On the other hand, the concentration polarization was also exacerbated with the increase of the sugar concentration. When the osmotic pressure was 16.0 bar, $J_{\mathrm{v}}$ declined from 108.0 to $67 \mathrm{~L} /\left(\mathrm{m}^{2} \mathrm{~h}\right)$, although the concentration of filtrated syrup ascended from 19.0 to $46.7 \mathrm{~g} / \mathrm{L}$.

\section{Effects of TMP on $R_{\text {obs }}$ of DP 6 to 8 COS}

As shown in Figure 4, the retentions of DP 6 to 8 COS varied with the TMP, and the $R_{\mathrm{obs}}$ of target oligosaccharides were significantly affected by the density and pressure applied. The influence of feed concentration on separation behaviors was emphasized at $28.3 \mathrm{~g} / \mathrm{L}$. Due to the existence of DP 6 to 8 COS, the increased concentrations were inevitably brought to a higher permeation of solutes. However, it was also found that the retention coefficient of DP 6 to 8 COS was immobile at higher-pressure areas (18.0 to 20.0 bar). The details could be explained by the steric hindrance interaction between solutes and membrane pores.

Additionally, the $R_{\text {obs }}$ of DP 6 to 8 COS increased with TMP, which indicate that the concentration polarization was a subordinate factor for the separation conditions in this case. As an alkaline molecule, the rejection properties of COS are significantly affected by the Donnan effects [23]. When the concentration was kept at $28.3 \mathrm{~g} / \mathrm{L}$, the $R_{\text {obs }}$ of solute decreased with the decrease of the pressure $(6.0$ to $16.0 \mathrm{bar}$ ). According to the Donnan theory, dielectric exclusion impels the increasing sugar accumulation, which is responsible for the considerable $R_{\text {obs }}$ of DP 6 to 8 COS. Therefore, it could be seen that the dropping of observed retention at a high-pressure range (18.0 to 20.0 bar) was similar. The corresponding results were of the same order with reported phenomena by Zhang [24]. Impressive retention proportions of DP 6 to $8 \mathrm{COS}$ at all selected concentrations and applied pressures were performed. 


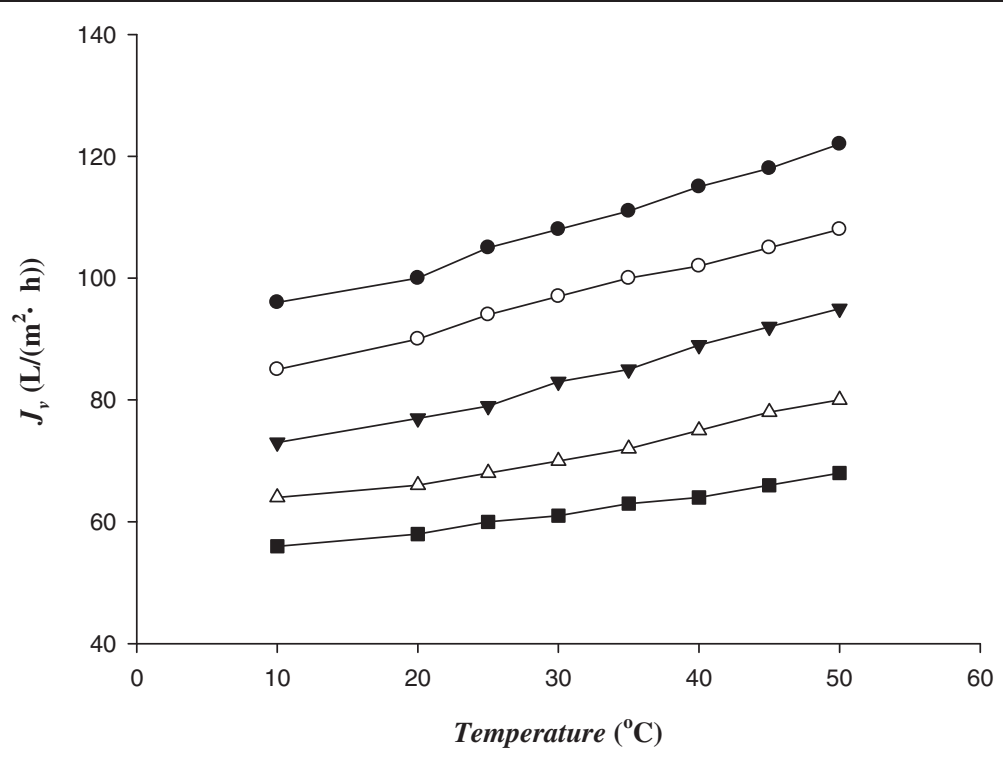

$\begin{array}{rr}\longrightarrow & 9.6 \mathrm{~g} / \mathrm{L} \text { DP } 6-8 \text { chitooligosaccharides } \\ \longrightarrow & 19.0 \mathrm{~g} / \mathrm{L} \text { DP } 6-8 \text { chitooligosaccharides } \\ \longrightarrow & 28.3 \mathrm{~g} / \mathrm{L} \text { DP } 6-8 \text { chitooligosaccharides } \\ \longrightarrow & 37.6 \mathrm{~g} / \mathrm{L} \text { DP } 6-8 \text { chitooligosaccharides } \\ \longrightarrow & 46.7 \mathrm{~g} / \mathrm{L} \text { DP } 6-8 \text { chitooligosaccharides }\end{array}$

Figure 5 Temperatures vs. $J_{v}$ of chitooligosaccharide syrup at $\mathbf{1 6 . 0}$ bar in NF process. It depicted the effects of $J_{v}$ on operation temperature, as well as the concentration variations of DP 6 to 8 chitooligosaccharides. Membrane pores were stretched by thermal expansion at high temperature, which reduced the rate of concentration polarization and improved the membrane flux.

\section{Effects of operation temperature on permeate flux}

Figure 5 illustrates the experimental data of membrane fluxes over operation temperature, as well as the concentration variations of DP 6 to 8 COS. It hypothesized that the syrup concentration was a constant and that the permeate flux during full recycle mode in the NF separation process was proportional to the temperature, which resulted from membrane swelling and the decrease of syrup viscosity under elevated temperature. As shown in Figure 5, the permeate flux was $79.0 \mathrm{~L} /\left(\mathrm{m}^{2} \mathrm{~h}\right)$ at $25^{\circ} \mathrm{C}$ when the concentration was set at $28.3 \mathrm{~g} / \mathrm{L}$. After the temperature rose to $40^{\circ} \mathrm{C}$, the permeate flux was correspondingly reached to $89.0 \mathrm{~L} /\left(\mathrm{m}^{2} \mathrm{~h}\right)$.

To be detailed, the membrane pores were stretched by structural modification when thermal expansion was interposed by external temperature. At the same moment, the increment of temperature meant for a decline of solution viscosity but a rise both in Reynolds number $(R e)$ and mass transfer coefficient $(k)$. Followed by the rules analyzed above, all solutes in the process preferred to move to the bulk part of the syrups, which reduced the rate of concentration polarization and thus improved the membrane flux.

However, excessive temperature was negative to preserve the stability of DP 6 to 8 COS. Because of the special structures that the amino group locates on $\mathrm{C}-2$ sites in every monomer unit linked by $\beta(1 \rightarrow 4)$ glycosidic bonds, the velocity of the Maillard reaction was motivated by high temperature. Certainly, further studies are needed to understand the mechanism of the Maillard reaction and its derivative products in permeate during the filtration process.

\section{Effects of operation temperature on $R_{\text {obs }}$ of DP 6 to $8 \mathrm{COS}$}

The retention manners of DP 6 to 8 chitooligosaccharides in different concentrations of syrup are shown in Figure 5, as the temperature varied from $10^{\circ} \mathrm{C}$ to $50^{\circ} \mathrm{C}$. As expected, the retentions of DP 6 to 8 chitooligosaccharides decreased with the increment of temperature. All curves coincide with the conclusion drawn from Figure 5. That is, the thermo swelling of membrane structures promoted more DP 6 to 8 chitooligosaccharides permeated through the NF membrane from the concentrate side. Specifically, the $R_{\text {obs }}$ of DP 6 to 8 chitooligosaccharides was 0.912 in $20^{\circ} \mathrm{C}$, whereas it diminished to 0.895 at $50^{\circ} \mathrm{C}$ with the concentration of $37.6 \mathrm{~g} / \mathrm{L}$. The transformation was apparently introduced that the $R_{\text {obs }}$ of solutes decreased with a boosting temperature that forced the fluid properties of 


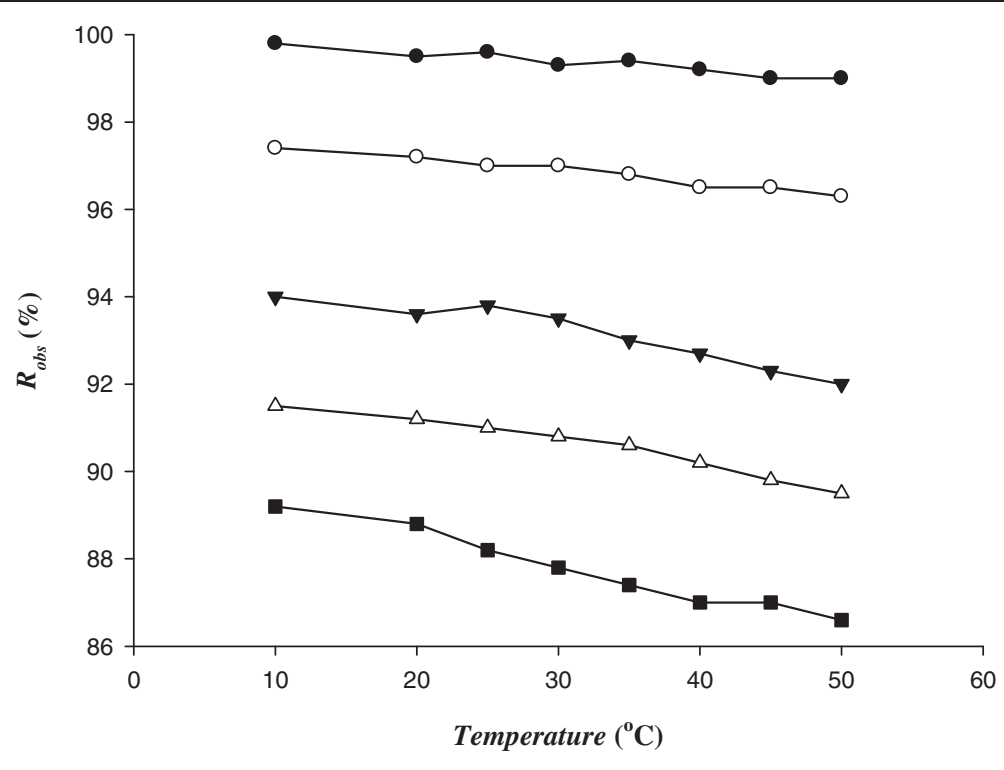

$\longrightarrow \quad 9.6$ g/L DP 6-8 chitooligosaccharides

- 19.0 g/L DP 6-8 chitooligosaccharides

$\longrightarrow \quad 28.3$ g/L DP 6-8 chitooligosaccharides

$\smile \_37.6 \mathrm{~g} / \mathrm{L}$ DP $6-8$ chitooligosaccharides

$\longrightarrow-46.7 \mathrm{~g} / \mathrm{L}$ DP $6-8$ chitooligosaccharides

Figure 6 Temperatures vs. $\boldsymbol{R}_{\text {obs }}$ of DP 6 to $\mathbf{8}$ chitooligosaccharides at $\mathbf{1 6 . 0}$ bar in NF process. It reflected the retention behaviors of DP 6 to 8 chitooligosaccharides in different concentrations when the temperature varied from $10^{\circ} \mathrm{C}$ to $50^{\circ} \mathrm{C}$. The retentions of DP 6 to 8 chitooligosaccharides decreased with the temperature increased.

chitooligosaccharide syrup to be a Newtonian liquid and hence resulted in a decrease in viscosity.

Besides, it is also worth noting that the charge effect shifted to become a major factor in the retention behaviors of DP 6 to 8 COS at variable concentrations (Figure 6). Under the same condition (i.e., TMP and temperature), the $R_{\text {obs }}$ of hexamer, heptamer, and octamer summed to 0.994 at a concentration of $9.6 \mathrm{~g} / \mathrm{L}$, but it declined to 0.874 at a concentration of $46.7 \mathrm{~g} / \mathrm{L}$. That could be explained by the Donnan steric pore model (DSPM) theory assumed by Bowen et al. [25]. Given that the negative charges were evenly distributed inside the selected membrane (QY-5-NF-1812), all of the opposite-charged ions as $\mathrm{H}^{+}$and $\mathrm{NH}_{4}^{+}$in the solution would be attracted thus the transport of COS was facilitated. Overall, the $J_{\mathrm{v}}$ and retention trends were both restricted by the concentration of syrup and its operation temperature.

\section{Effects of $\mathrm{pH}$ on $R_{\mathrm{obs}}$ of $\mathrm{COS}$}

The effects of pH ranged from 5.0 to 9.0 on rejections of glucosamine and DP 2 to 8 COS were investigated. Singles situated in the area of 13.0 to $19.0 \mathrm{~min}$ represent COS within DP 6 to 8. As shown in Figure 7, the $R_{\text {obs }}$ of COS increased with $\mathrm{pH}$, especially for the monomer, dimer, trimer, and tetramer. In the chromatogram of NF concentrate at $\mathrm{pH}$ 5.0, it was found that nearly all of COS (DP $\leq 4)$ had permeated through the membrane, whereas higher DP were effectively rejected. On the contrary, when the syrup was adjusted to a basic condition $(\mathrm{pH}=9.0)$, the $R_{\text {obs }}$ of the monosaccharide and disaccharides arrived at 0.458 and 0.864 , respectively, even that the trimer and tetramer completely escaped from the permeate. The reason for this phenomenon might be due to the inter- and intramolecular hydrogen bonds, which further resulted in the unequal curling and overlap in structures. Similar results on $R_{\text {obs }}$ and inference for COS have been taken by Han et al. [20]. The stability of the primary structure was broken by hydrogen bonds and restabilized spontaneously. During the recombination process, the curling extent would extremely impact the viscosity and stereospecific blockage of molecules. The structural explosion guided the poor affinity of solutes with the membrane and led to a high resistance of permeation. Furthermore, the rejections of DP 6 to 8 COS, formulated from the concentrate and permeate profiles, were scarcely impacted via $\mathrm{pH}$ options (Figure 7). The results indicated that the applied driving force was centered on oligomers of DP $\leq 4$ under acidic conditions and sequentially spread to higher polymers as the $\mathrm{pH}$ increased. DP 6 to $8 \mathrm{COS}$ is expected to be completely rejected by the NF membrane at a wider 

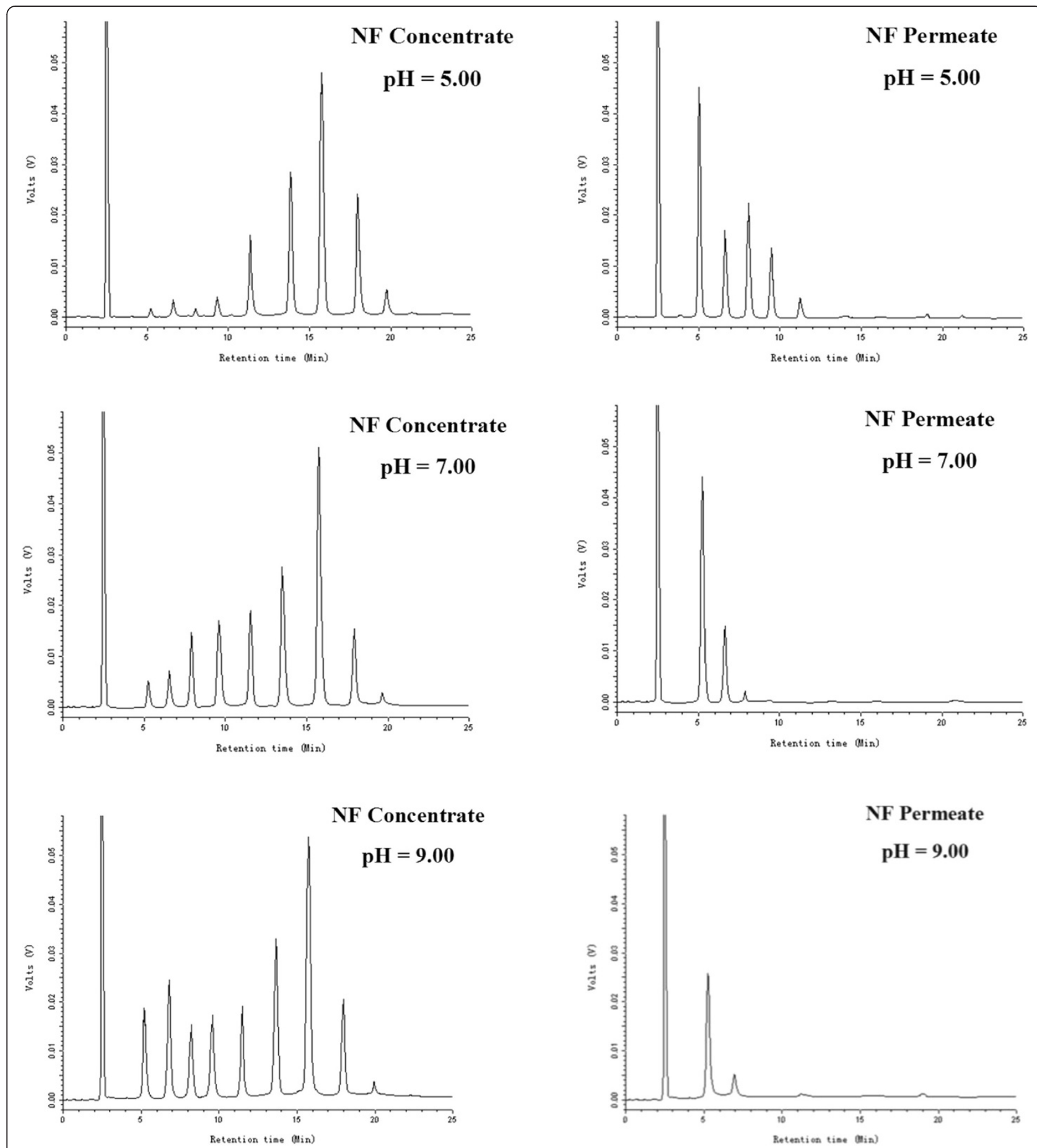

Figure $7 \mathrm{pH}$ values vs. $\boldsymbol{R}_{\text {obs }}$ of chitooligosaccharides at 16.0 bar and $40^{\circ} \mathrm{C}$ in NF process. The effects of pH ranged from 5.0 to 9.0 on rejections of glucosamine and DP 2 to 8 chitooligosaccharides were investigated. The $R_{\text {obs }}$ of chitooligosaccharides increased with pH, especially for the monomer, dimer, trimer, and tetramer. In addition, the flexibility of chitooligosaccharides was well conducted by $\mathrm{pH}$.

$\mathrm{pH}$ range (5.0 to 9.0). More important, the flexibility of COS was well conducted by the adjustment of $\mathrm{pH}$. Nevertheless, in view of the objective of purification, a lower $\mathrm{pH}$ should be adopted for the impurity elimination. Interestingly, several small-sized peaks that were followed by octasaccharides were also characterized at $19.5 \mathrm{~min}$ (Figure 7). Due to the randomness of enzymatic hydrolysis on chitosan, this was probably attributed to the nonamers (DP 9 COS) existing in the syrup. 


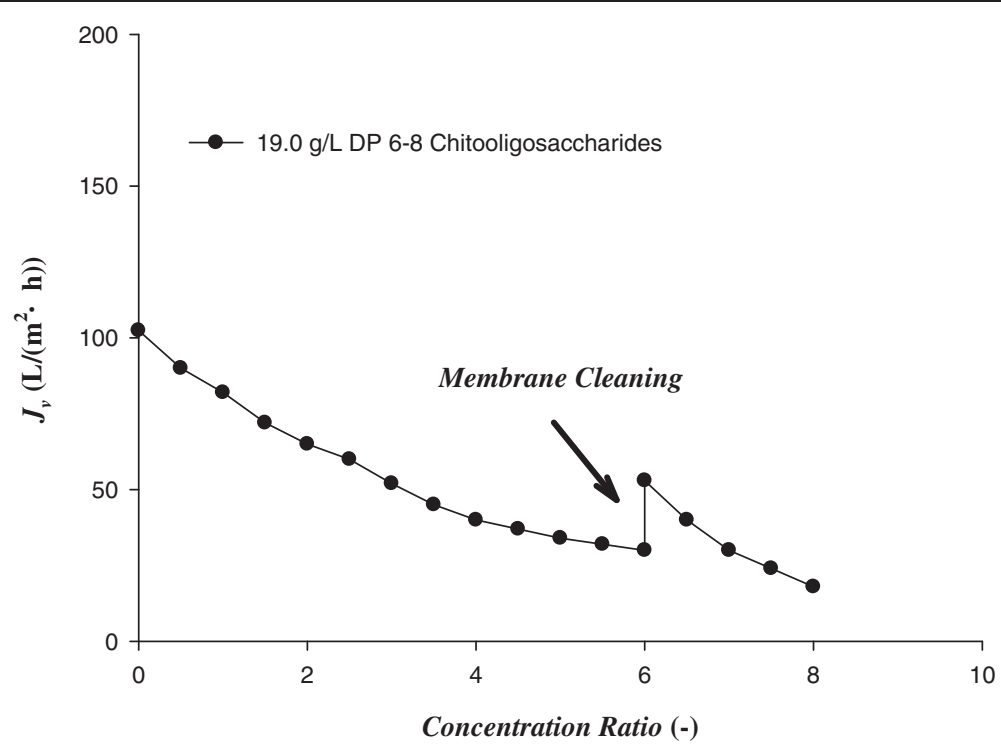

Figure 8 Concentration ratios vs. $J_{\mathrm{v}}$ of $19.0 \mathrm{~g} / \mathrm{L}$ chitooligosaccharide syrup. Long-term experiments were carried out at 16.0 bar for enrichment of DP 6 to 8 chitooligosaccharides. The flux was about $102.4 \mathrm{~L} /\left(\mathrm{m}^{2} \mathrm{~h}\right)$ at the beginning, whereas it decreased heavily when the concentration rate was 6.0.

\section{Purification of DP 6 to 8 COS}

Long-term experiments were carried out at 16.0 bar to enrich the DP 6 to 8 COS with a virgin concentration of $19.0 \mathrm{~g} / \mathrm{L}\left(\mathrm{pH}=5.0, \mathrm{~V}_{0}=16.0 \mathrm{~L}\right.$ and $\left.\mathrm{T}=40^{\circ} \mathrm{C}\right)$. The performance of the NF membrane was evaluated by measuring the permeate flux and $R_{\text {obs }}$ of the target products as illustrated in Figures 8 and 9. In addition, the concentration ratio of DP 6 to 8 COS was monitored as a formula of $V_{0} / V_{\mathrm{c}}$. The flux value was about $102.4 \mathrm{~L} /\left(\mathrm{m}^{2} \mathrm{~h}\right)$ at the beginning of the experiment, but it decreased to $30.2 \mathrm{~L} /\left(\mathrm{m}^{2} \mathrm{~h}\right)$ when the concentration ratio reached to 6.0 (Figure 8). Then, the membrane was cleaned for $1 \mathrm{~h}$. After that, the permeate flux of syrup was recovered to $53.5 \mathrm{~L} /\left(\mathrm{m}^{2} \mathrm{~h}\right)$, which indicated that about $50 \%$ of virtual flux decline due to the increasing solid content and viscosity. However, the second drop of permeate flux was remarkable till the end of the run, as the value changed from 53.5 to $25.0 \mathrm{~L} /\left(\mathrm{m}^{2} \mathrm{~h}\right)$ just 1.5 times of the later concentration. There was a direct reflection between the effect of solid contents and experimental records. A moderate concentration ratio and timely

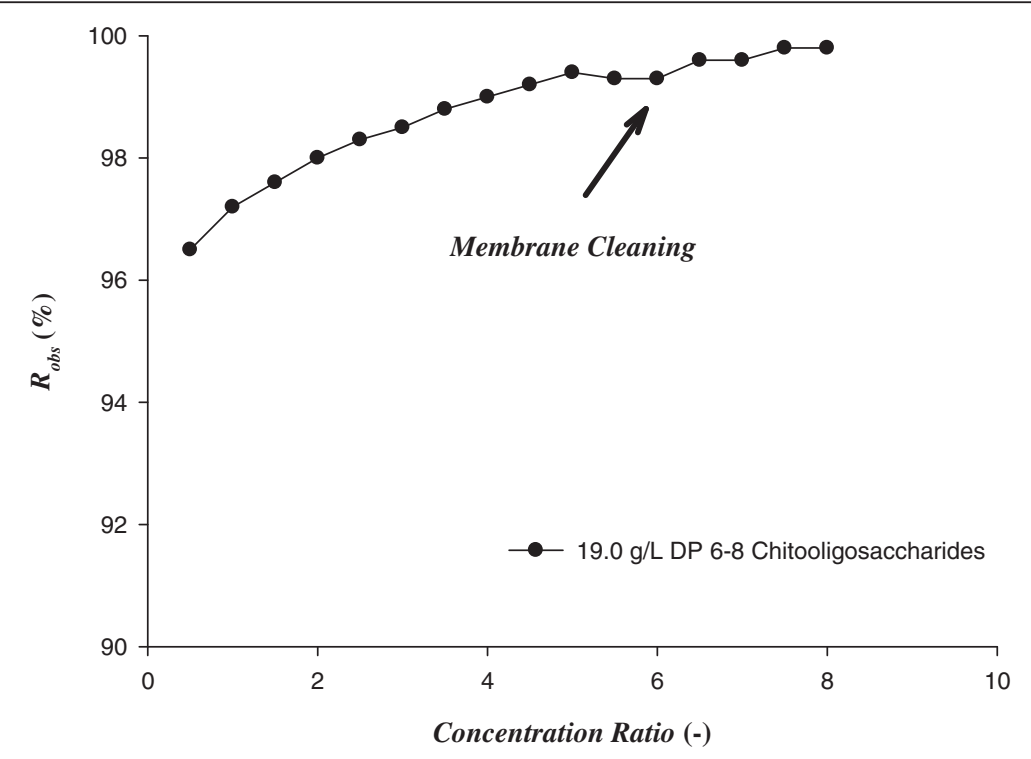

Figure 9 Concentration ratios vs. $\boldsymbol{R}_{\text {obs }}$ of DP 6 to 8 chitooligosaccharides in the syrup. The $R_{\text {obs }}$ of DP 6 to 8 chitooligosaccharides went up when the circulating flux decreased. The phenomenon was accredited to the DSPM and steric hindrance pore (SHP) effects. 


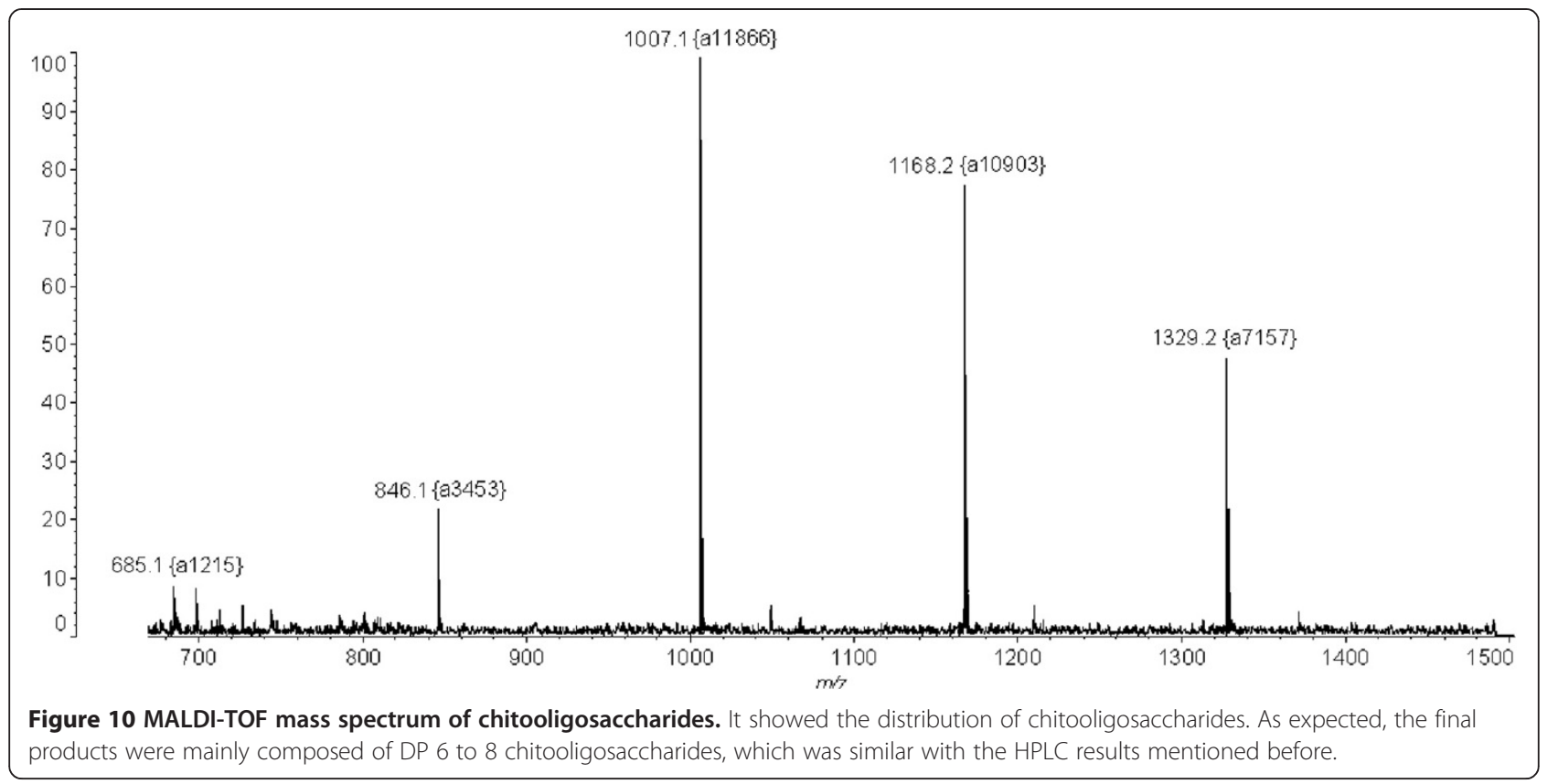

regenerations of the membrane should be arranged for acceptable efficiency in practice.

Moreover, the $R_{\text {obs }}$ of DP 6 to 8 COS went up when the circulating flux decreased (Figure 9). The result was principally accredited to the synergy with the DSPM and steric hindrance pore (SHP) effects. On the one hand, the anions preferred to be repelled while the cations were attracted to the NF membrane. The unique property transported the amino groups, which are commonly sensed in COS, to the permeate. Ultimately, the molecules containing $\mathrm{NH}_{4}^{+}$ were separated from the syrup because of the electric attraction. On the other hand, the $R_{\mathrm{obs}}$ of DP 6 to 8 COS was up to 1.0 after the concentration ratio increased to 8.0. The MWCO of the applied membrane is $500 \mathrm{Da}$, while the molecular weights of hexamer, heptamer, and octamer are 984, 1,145 and 1,306, respectively. There is a conspicuous difference in the sizes between membrane pores and DP 6 to 8 COS. Therefore, the observed retention behaviors illustrated that DP 6 to 8 COS were rejected sterically and a promising purity (82.2\%) was eventually achieved via the NF purification designed.

\section{MALDI-TOF-MS analysis of chitooligosaccharides}

Matrix-assisted laser desorption/ionization time-of-flight (MALDI-TOF) is an outstanding tool for the comprehensive investigation of COS because the mass spectrum can exhibit the relative quantities of a mixture to be determined. Figure 9 shows the distribution of COS using MALDI-TOF-MS after NF enrichment. As expected, the final products were mainly composed of DP 6 to 8 COS, which was similar to the HPLC results mentioned before. In the spectrum, COS often contains intensive quasi-molecular ions, which is called $[\mathrm{M}+\mathrm{Na}]^{+}$, because of its weak protonation degree. For instance, the peak at $1,007.1 \mathrm{~m} / \mathrm{z}$ is attributed to the sodium form $\left([\mathrm{M}+\mathrm{Na}]^{+}\right)$ of a hexa-oligomer (Figure 10).

\section{Conclusions}

In this study, the separation behavior of COS syrups, which were enriched by different concentrations of DP 6 to $8 \mathrm{COS}$, was investigated via a bench-scale NF process.

One negatively charged membrane with MWCO of 500 Da was selected to purify DP 6 to 8 COS. During the full recycle mode, the experimental results indicated that the retentions of these components increased with pressure before 16.0 bar. Also, the operation temperature was optimized. Although the circulating flux could be improved by elevating temperature, a greater wastage of DP 6 to $8 \mathrm{COS}$ was irreversibly formed during the process as well. In addition, the effects of $\mathrm{pH}$ on $R_{\mathrm{obs}}$ of COS were compared. The HPLC profiles illustrated that the $R_{\text {obs }}$ of COS within DP $\leq 4$ in alkali conditions was significantly higher than that in acidic environment. This phenomenon could be explained by structural curling and sterical overlap due to hydrogen bonds. However, the mechanisms should be discussed in further researches.

Under the optimum conditions (TMP $=16.0$ bar, $T=40^{\circ} \mathrm{C}$, and $\mathrm{pH}=5.0$ ), the purification of DP 6 to 8 $\mathrm{COS}$ was carried out. It was found that the membrane could support a reluctant flux after the concentration ratio was over 6.0 in the syrup with the concentration of $19.0 \mathrm{~g} / \mathrm{L}$ DP 6 to 8 COS. MALDI-TOF mass spectrum confirmed that DP 6 to 8 COS were dominant in the final products, and the purity was up to $82.2 \%(w / w)$ 
according to HPLC profiles. As a conclusion, the NF system equipped with a selected membrane module is a promising approach in the purification of DP 6 to 8 COS from specific syrups.

\section{Competing interests}

The authors declare that they have no competing interests.

\section{Authors' contributions}

This paper is the result of joint efforts. Prof. LZ designed the whole experimental plan and confirmed the main objective of this paper. Dr. YW developed the statistical methods for experimental data. HD was responsible for optimization of the nanofiltration technology and partial investigation of the transmechanism in process. QX was responsible for the quantification of proteins and total sugars. HPLC and MALDI-TOF-MS analysis were done by Prof. JZ and Prof. LJ. LF helped us complete the paper writing and correcting some grammatical errors in details. All authors read and approved the final manuscript.

\section{Acknowledgements}

This work is financially supported by the National Natural Science Foundation of China (No. 31371725 and No. 31101381). Also, the authors are grateful to the Fundamental Research Funds for the Central Universities.

Received: 13 July 2014 Accepted: 14 October 2014

Published online: 19 November 2014

\section{References}

1. Xia W, Liu P, Zhang J, Chen J (2011) Biological activities of chitosan and chitooligosaccharides. Food Hydrocolloids 25:170-179

2. Kim S, Rajapakse N (2005) Enzymatic production and biological activities of chitosan oligosaccharides (COS): a review. Carbohydr Polym 62:357-368

3. Benhabiles MS, Salah R, Lounici H, Drouiche N, Goosen MFA, Mameri N (2012) Antibacterial activity of chitin, chitosan and its oligomers prepared from shrimp shell waste. Food Hydrocolloids 29:48-56

4. Huang RH, Du YM, Yang JH, Fan L (2003) Influence of functional groups on the in vitro anticoagulant activity of chitosan sulfate. Carbohy Res 338:483-489

5. Lin S, Lin Y, Chen H (2009) Low molecular weight chitosan prepared with the aid of cellulase, lysozyme and chitinase: characterisation and antibacterial activity. Food Chem 116:47-53

6. Kim KW, Thomas RL (2007) Antioxidative activity of chitosans with varying molecular weights. Food Chem 101:308-313

7. Zhang Y, Huo M, Zhou J, Yu D, Wu Y (2009) Potential of amphiphilically modified low molecular weight chitosan as a novel carrier for hydrophobic anticancer drug: synthesis, characterization, micellization and cytotoxicity evaluation. Carbohydr Polym 77:231-238

8. Zhang J, Zhang W, Mamadouba B, Xia W (2012) A comparative study on hypolipidemic activities of high and low molecular weight chitosan in rats. Int J Biol Macromol 51:504-508

9. Chang Y, Chang C, Huang T, Chen S, Lee J, Chung Y (2011) Effects of low molecular weight chitosans on aristolochic acid-induced renal lesions in mice. Food Chem 129:1751-1758

10. Berth G, Dautzenberg H (2002) The degree of acetylation of chitosans and its effect on the chain conformation in aqueous solution. Carbohydr Polym 47:39-51

11. Lee EH, Lee JJ, Jon SY (2010) A novel approach to oral delivery of insulin by conjugating with low molecular weight chitosan. Bioconjugate Chem 21:1720-1723

12. Li N, Wang CY, Wang M, Sun X, Nie S, Pan W (2009) Liposome coated with low molecular weight chitosan and its potential use in ocular drug delivery. Int J Pharm 379:131-138

13. Chien PJ, Sheu F, Lin HR (2007) Coating citrus (Murcott tangor) fruit with low molecular weight chitosan increases postharvest quality and shelf life. Food Chem 100:1160-1164

14. Abd-Elmohdy FA, Sayed ZE, Essam S, Hebeish A (2010) Controlling chitosan molecular weight via bio-chitosanolysis. Carbohydr Polym 82:539-542

15. Jeon Y, Kim S (2000) Production of chitooligosaccharides using an ultrafiltration membrane reactor and their antibacterial activity. Carbohydr Polym 41:133-141
16. Fan $\mathrm{W}$, Yan $\mathrm{W}, \mathrm{Xu}$ Z, Ni H (2012) Formation mechanism of monodisperse, low molecular weight chitosan nanoparticles by ionic gelation technique. Colloids Surf B 90:21-27

17. Cabrera JC, Custem PV (2005) Preparation of chitooligosaccharides with degree of polymerization higher than 6 by acid or enzymatic degradation of chitosan. Biochem Eng J 25:165-172

18. Simonnot MO, Castel C, Nicolai M, Rosin C, Sardin M, Jauffret H (2000) Boron removal from drinking water with a boron selective resin: is the treatment really selective. Water Res 34:109-116

19. Nadav N (1999) Boron removal from seawater reverse osmosis permeate utilizing selective ion exchange resin. Desalination 124:131-135

20. Han YP, Lin Q, He XW (2009) Research on desalination and purification characteristics of chitobiose solution with nanofiltration membrane. Membrane Science and Technology 29:105-109

21. Han YP, Lin Q, Wang XL (2012) Feasibility study on chitooligosaccharides purification by nanofiltration membranes. Ion Exchange and Adsorption 28:86-96

22. Xu W, Jiang C, Kong X, Liang Y, Rong M, Liu W (2012) Chitooligosaccharides and $\mathrm{N}$-acetyl-D-glucosamine stimulate peripheral blood mononuclear cell-mediated antitumor immune response. Mol Med Rep 6:385-390

23. Zhao HF, Hua X, Yang RJ, Zhao LM, Zhao W, Zhang Z (2012) Diafiltration process on xylo-oligosaccharides syrup using nanofiltration and its modeling. Int J Food Sci Tech 47:32-39

24. Zhang Z, Yang RJ, Zhang S, Zhao H, Hua X (2011) Purification of lactose syrup by using nanofiltration in a diafiltration mode. J Food Eng 105:112-118

25. Bowen WR, Mohammad AW (1998) Diafiltration by nanofiltration: prediction and optimization. AlChE J 44:1799-1812

doi:10.1186/s40643-014-0020-x

Cite this article as: Dong et al:: Purification of DP 6 to 8

chitooligosaccharides

by nanofiltration from the prepared chitooligosaccharides syrup

Bioresources and Bioprocessing 2014 1:20.

\section{Submit your manuscript to a SpringerOpen ${ }^{\circ}$ journal and benefit from:}

- Convenient online submission

- Rigorous peer review

- Immediate publication on acceptance

- Open access: articles freely available online

- High visibility within the field

- Retaining the copyright to your article

Submit your next manuscript at $>$ springeropen.com 